\title{
miR-668 inhibitor attenuates mitochondrial membrane potential and protects against neuronal apoptosis in cerebral ischemic stroke
}

\author{
Junna $\mathrm{He}^{1}$, Xiangjian Zhang ${ }^{2}$ \\ ${ }^{1}$ Department of Neurology, Hebei Medical University, Shijiazhuang, Hebei, China, ${ }^{2}$ Department of Neurology, The Second Hospital \\ of Hebei Medical University, Shijiazhuang, Hebei, China
}

\begin{abstract}
Cerebral stroke is a major cause of brain injury due to the production of hypoxic conditions, and new therapeutic interventions are required for its management. Here, we evaluated the protective effect of miR-668 inhibitor against ischemia/reperfusion (I/R)-induced stroke. Cerebral stroke was induced by cerebral artery occlusion in rats, followed by treatment with miR-668 inhibitor for 10 minutes before reperfusion. The neuroprotective effect of miR-668 inhibitor was determined by estimating the neurological deficit score, cerebral infarct area and blood-brain barrier (BBB) permeability. In addition, the levels of inflammatory cytokines, and the expression of NLRP3, zonula occludens- 1 (ZO-1), dynamin-related protein 1 (Drp1) and occludin proteins, were estimated by ELISA and Western blotting, respectively. TUNEL assay and immunohistochemical analyses were performed to examine the effects of miR-668 inhibitor against I/R-induced stroke rats. The miR-668 inhibitor treatment group showed reductions in the infarct area, $B B B$ permeability and neurological score compared to the stroke group. The levels of cytokines and reactive oxygen species were reduced in the miR-668 inhibitor treatment group compared to the stroke group. These observations suggested that inhibition of miR-668 reduces neuronal apoptosis by ameliorating the expression of caspase 3 , Bax and BCl-2 protein in 1/R stroke rats. The expression of NLRP3, ZO-1 and occludin proteins was attenuated in the brain tissue of the miR-668 inhibitor treatment group compared to the stroke group. Moreover, the phosphorylation of Drp 1 protein was reduced in the miR-668 inhibitor group compared to the stroke group. In conclusion, the results of the present study indicated that inhibition of miR-668 prevented neuronal apoptosis in cerebral I/R-induced stroke by modulating mitochondrial function and regulating NLRP3 signalling.
\end{abstract}

Key words: miR-668 inhibitor, cerebral stroke, neuroprotection, apoptosis, mitochondria, inflammation.

\section{Introduction}

Cerebral ischemia is a major cause of physical disability and mortality worldwide [19]. Reperfusion after the management of ischemia leads to secondary cerebral injury, commonly known as ischemia/ reperfusion (I/R) injury [10]. Several pathways are involved in the development of secondary injury, including autophagy, apoptosis, calcium overload, excitotoxicity, oxidative stress, impaired energy production and inflammation [4]. The current approach to management of secondary injury due to cerebral I/R-induced neuronal injury has several limitations. Autophagy in neuronal cells is regulated by the inflammatory cascade via inflammasomes. 
Inflammation and immunity are regulated by inflammasomes, and NLRP3 is a major intracellular multiprotein complex that recognises pathogen-associated molecular patterns [20]. NLRP3 enhances the inflammatory response by activating cytokines and caspase 1 at sites of injury [11]. NLRP3 is involved in the development of a number of disorders, including Alzheimer's disease, atherosclerosis, cancer and diabetes [16]. Moreover, NLRP3 is involved in the development of cerebral I/R-induced neuronal injury [15].

There is an imbalance in the production and utilisation of brain energy in cerebral ischemia, which leads to neuronal injury due to cerebral oedema, neuronal oedema and inflammation [18]. The impairment of cellular energy production causes mitochondrial dysfunction in neuronal cells [27]. Mitochondrial fission is promoted by the activation of dynamin-related protein 1 (Drp1) [13]. Under physiological conditions, Drp1 is present only in the mitochondria fraction [5]. Phosphorylation of Drp1 reduces mitochondrial fission by regulating its translocation via cyclic adenosine monophosphate-dependent protein kinase (AMPK) [6]. Neuronal apoptosis is stimulated by altering membrane permeability, through the fragmentation and fission of mitochondria [3].

microRNAs (miRNAs) are a group of small noncoding RNA molecules (22 nucleotides in length) that regulate gene expression at the post-transcriptional level. The expression profiles of miRNAs in normal cells and tissue are fixed, but have been reported to be altered in neurological disorders [14]. The expression level of the miRNA, miR-668, was reported to be altered under ischemic conditions in cell culture and animal models [2]. The repression of miR-668 was suggested to prevent the apoptosis of nephrons in acute kidney injury, by preventing mitochondrial fragmentation and repression [25]. The present study was performed to evaluate the potential effect of miR-668 inhibition on neuronal apoptosis in cerebral ischemic stroke.

\section{Material and methods}

\section{Animals}

Male Sprague-Dawley rats weighing 250-300 g were kept under a 12-hour light/dark cycle (humidity, $60 \pm 5 \%$; temperature, $24 \pm 3^{\circ} \mathrm{C}$ ). The protocols used in the animal experiments were approved by the Institutional Animal Ethical Committee of The
Second Hospital of Hebei Medical University, China (IAEC/SH-HMU/2017/06).

\section{Chemicals}

miR-668 inhibitor was purchased from Sigma Aldrich (St. Louis, MO, USA). Enzyme-linked immunosorbent assay (ELISA) kits were purchased from R\&D Systems (Minneapolis, MN, USA). Antibodies used in western blotting assay were obtained from Thermo Fisher Scientific (Wilmington, DE, USA).

\section{Experiments}

Ischemia/reperfusion neuronal injury was induced in a middle cerebral artery (MCA) occlusion model, as reported previously [23]. The left external and common carotid arteries were exposed and ligated in anaesthetised animals. A monofilament nylon suture was used to ligate the MCA. The monofilament was inserted until it reached the origin of the MCA and local cerebral blood flow decreased to $<16 \%$ of the baseline level. Reperfusion involved restoring the cerebral blood flow to $75 \%$ after 1 hour of ischemia by releasing the ligature.

Animals were divided into three groups: sham group; I/R-induced stroke group; and the miR-668 inhibitor treatment group, which received miR-668 inhibitor for 10 minutes before reperfusion.

\section{Neurological dysfunction}

Neurological dysfunction was assessed by determining neurological scores in I/R-induced neuronal injury rats using Longa's method [26], as follows: 0 - no deficit; 1 - forelimb failed to extend entirely and weak; 2 - circling to the contralateral side; 3 - weight-bearing capacity reduced on the injured side; and 4 - no spontaneous locomotor activity.

\section{Estimation of cerebral infarction}

The brain was isolated after 1 day of reperfusion, and 2 -mm-thick coronal brain sections were stained for 30 minutes at $37^{\circ} \mathrm{C}$ with $2 \%$ 2,3,5-triphenyltetrazolium chloride. Image J software $(\mathrm{NIH}$, Bethesda, MD, USA) was used to determine the percentage of infarct area based on photographs of the slices.

\section{Estimation of BBB permeability}

The permeability of the blood-brain barrier (BBB) was estimated by examining the extravasation of Evans Blue (EB) into the brain. Animals were inject- 
ed with $2 \%$ EB at a dose of $2 \mathrm{ml} / \mathrm{kg}$ via the tail vein immediately after reperfusion. The brain was dissected from each animal after sacrifice, and the brain tissue was homogenised in phosphate-buffered saline (PBS). The supernatant of the homogenate was separated by centrifugation for 30 minutes at 15000 rpm. The supernatant was diluted with trichloroacetic acid solution (50\%), followed by incubation for 2 hours at room temperature. A spectrophotometer was used to determine the absorbance of the supernatant at 610 nm (UV-160A; Shimadzu, Kyoto, Japan).

\section{Estimation of cytokines}

The levels of the inflammatory mediators inter-

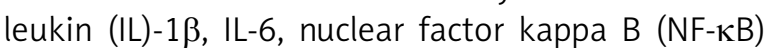
and tumour necrosis factor $\alpha$ (TNF- $\alpha$ ) were determined in the brain tissue homogenates by ELISA using commercial kits in accordance with the manufacturer's protocols (R\&D Systems).

\section{Estimation of ROS}

MitoSOX red mitochondrial superoxide indicator was used to estimate the levels of reactive oxygen species (ROS) in the brain tissues. Briefly, tissue homogenates were stained at $37^{\circ} \mathrm{C}$ in the dark for 30 minutes with $5 \mu \mathrm{M}$ MitoSOX red. A fluorescent plate reader was used to estimate the intracellular ROS levels at excitation and emission wavelengths of 510 and $580 \mathrm{~nm}$, respectively.

\section{Western blotting}

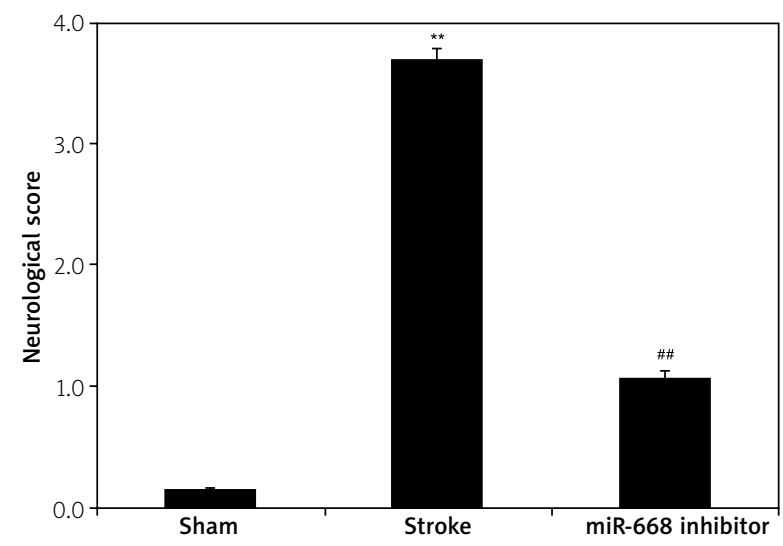

Fig. 1. miR-668 inhibitor attenuates the neurological functions in the $1 / R$ induced stroke rat model. Mean \pm SEM $(n=6),{ }^{\# \#} p<0.01$ compared to the sham group; ${ }^{* *} p<0.01$ compared to the stroke group.
The protein expression levels of Bax, Bcl-2, caspase-3, p-Drp1, Drp1, NLRP3, zonula occludens-1 (ZO-1) and occludin were assessed by Western blotting analysis of brain tissue homogenates. A BCA assay kit was used to quantify the protein from each tissue homogenate, and $10 \%$ sodium dodecyl sulphate-polyacrylamide gel electrophoresis (SDSPAGE) was performed to separate the proteins, which were electroblotted onto nitrocellulose membranes. Subsequently, each membrane was blocked with $5 \%$ blocking solution (non-fat milk) and incubated in blocking buffer with primary antibodies overnight at $4^{\circ} \mathrm{C}$. Goat secondary antibodies conjugated with horseradish peroxidase were added to the blocking buffer, and a chemiluminescence kit was used to detect the proteins.

\section{TUNEL assay}

Terminal deoxynucleotidyl transferase dUTP nick end labelling (TUNEL) assay was used to determine apoptosis of myocardial cells. The apoptosis index was calculated as follows: no. of stained myocytes/ total no. of myocytes $\times 100 \%$.

\section{Statistical analysis}

All data are expressed as means \pm standard error of the mean (SEM; $n=6$ ) and the statistical analysis consisted of one-way analysis of variance (ANOVA). Post hoc comparisons were carried out with Dunnett's test using GraphPad Prism software (ver. 6.1; GraphPad Software, Inc., San Diego, CA, USA). In all analyses, $p<0.05$ was taken to indicate statistical significance.

\section{Results \\ miR-668 inhibitor attenuates neurological functions}

The effects of miR-668 inhibition on neurological function score were determined in the I/R-induced stroke rat model. The neurological score was increased to 3.7 in the stroke group, while the score was only 0.1 in the sham group. The miR-668 inhibitor treatment group showed a reduction in neurological score to $1.07 \mathrm{com}$ pared to the stroke group (Fig. 1). These observations indicated that inhibition of miR-668 improved neurological function by reducing the neurological score in the I/R-induced stroke rat model.

miR-668 inhibitor attenuates 
A

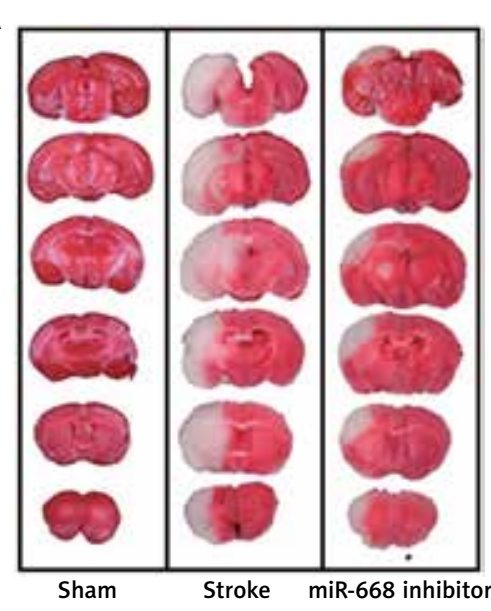

B 35.0

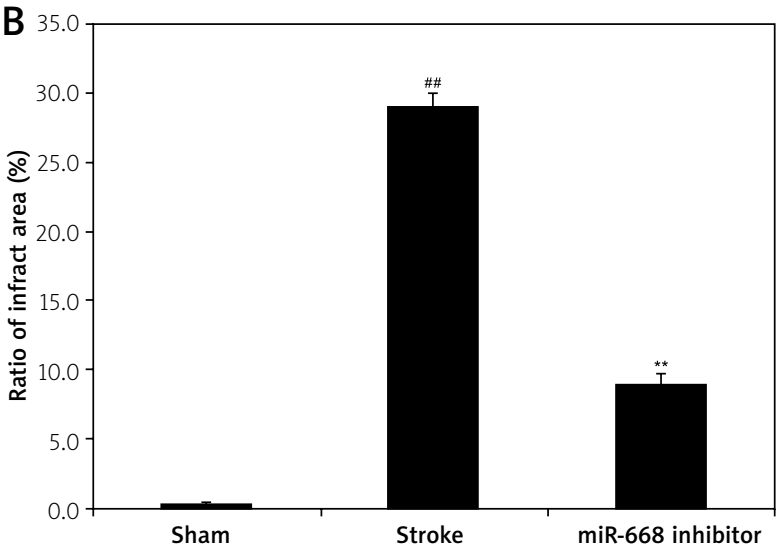

Fig. 2. miR-668 inhibitor attenuates the percentage of the cerebral infracted area in the I/R induced stroke rat model. Mean \pm SEM $(n=6),{ }^{\# \#} p<0.01$ compared to the sham group; ${ }^{* *} p<0.01$ compared to the stroke group.

\section{the increase in infarct area}

The effects of miR-668 inhibitor treatment on the cerebral infarct area were examined in the I/R-induced stroke rat model (Fig. 2). The percentage of infarct area was increased in the stroke group (29\%) compared to the sham group (0.3\%). However, inhibition of miR-668 in the I/R-induced stroke rat model significantly reduced the infarct area to $9 \%$ $(p<0.01)$.

\section{miR-668 inhibitor attenuates BBB permeability}

The permeability of the BBB was assessed by determining the amount of EB present in the brain tissue of miR-668 inhibitor-treated I/R-induced stroke rats. An increase was observed in the amount of EB in the brain tissue of the stroke group, indicating increased BBB permeability in these rats. However, treatment with miR-668 inhibitor reduced the amount of $E B$ in the brain tissue, indicating improvement in BBB permeability (Fig. 3).

\section{miR-668 inhibitor attenuates the increase in cytokine levels}

Figure 4 shows the levels of cytokines, i.e. IL-1 $\beta$, $N F-\kappa B$, TNF- $\alpha$ and IL-8, in the brain tissue of I/R-induced stroke rats. The levels of cytokines were increased in the neuronal tissues of the stroke group compared to the sham-operated controls. The miR-

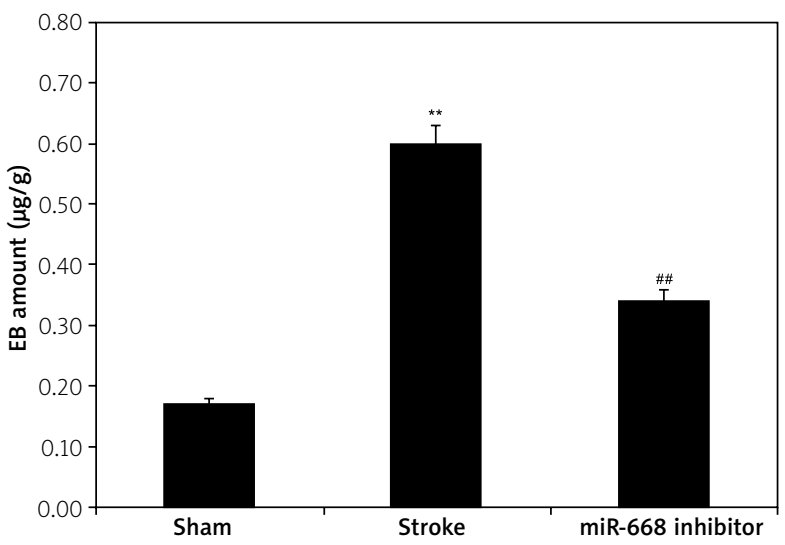

Fig. 3. miR-668 inhibitor attenuates the BBB permeability in the I/R induced stroke rat model.

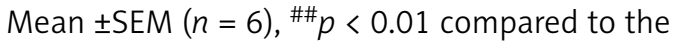
sham group; ${ }^{* *} p<0.01$ compared to the stroke group.

668 inhibitor treatment group showed reduced cytokine levels compared to the sham group.

\section{miR-668 inhibitor attenuates neuronal cell apoptosis}

The effect of miR-668 inhibition on the apoptosis of neurons was examined by determining the number of TUNEL-positive cells and Bcl-2, Bax and caspase-3 protein expression levels in the cerebral tissue of I/R-induced stroke model rats (Fig. 5). The apoptosis index was higher in the cerebral tissue of the stroke group compared to the sham group. The apoptosis index was 


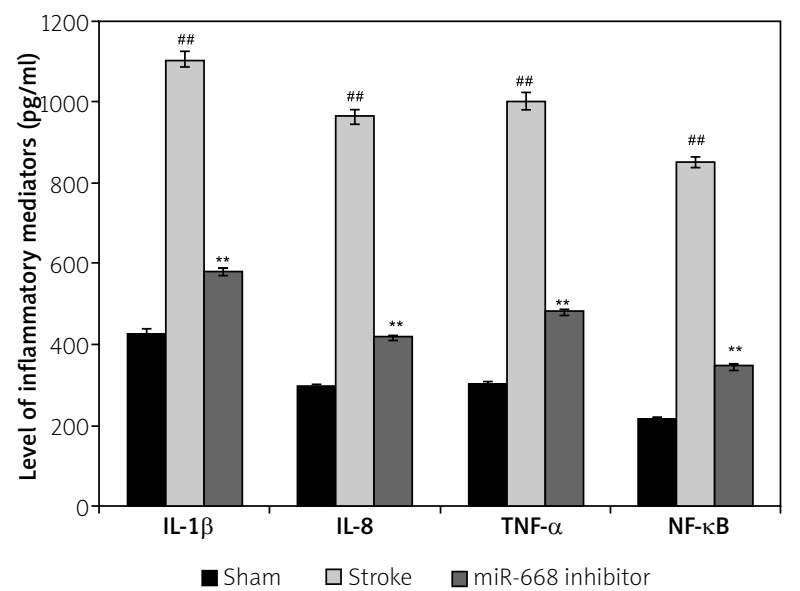

Fig. 4. miR-668 inhibitor attenuates the level of cytokines in the I/R induced stroke rat model.

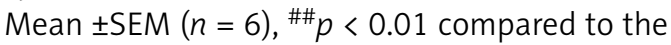
sham group; ${ }^{* *} p<0.01$ compared to the stroke group. reduced in the miR-668 inhibitor group compared to the sham group (Fig. 5A). The levels of Bax, caspase-3 and Bcl-2 protein expression were estimated by Western blotting analysis as markers of neuronal apoptosis. Both Bax and caspase 3 protein expressions were significantly increased $(p<0.01)$, while Bcl-2 protein expression was reduced, in the cerebral tissue of the stroke group compared to the sham-operated control group. Treatment with miR-668 inhibitor ameliorated the alterations in Bax, caspase- 3 and $\mathrm{Bcl}-2$ protein levels in the cerebral tissue of I/R-induced stroke rats (Fig. 5B).

\section{miR-668 inhibitor attenuates NLRP-3 signalling}

The effects of miR-668 inhibitor on the NLRP3 signalling pathway were examined by determining the ZO-1, NLRP3 and occludin protein expression levels in I/R-induced stroke rats, as shown in Figure 6.

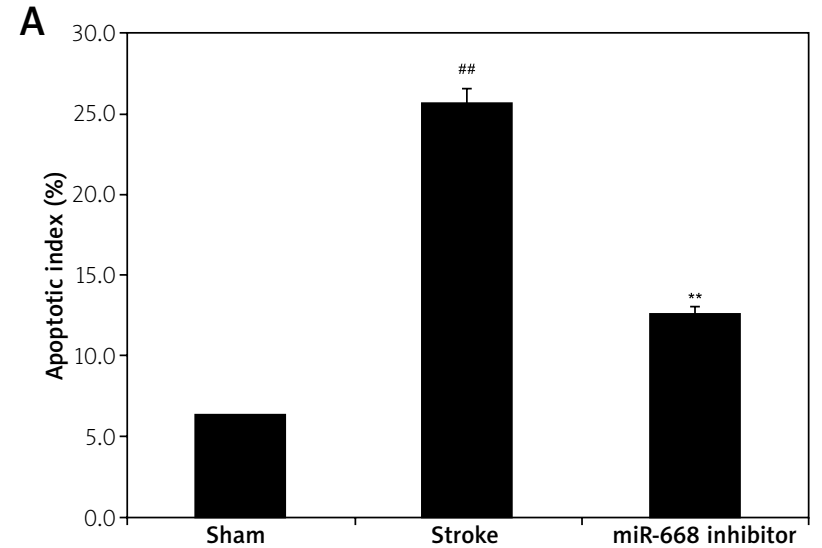

B
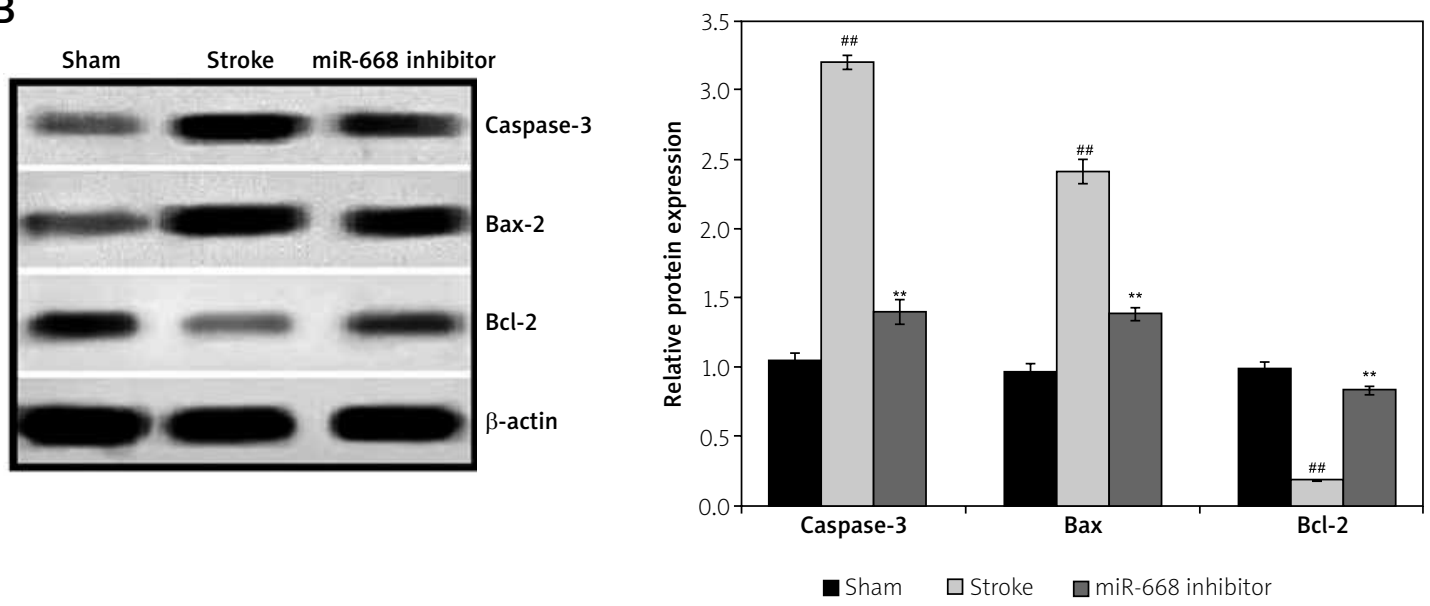

Fig. 5. miR-668 inhibitor attenuates the apoptosis of neuronal cells in the I/R induced stroke rat model. A) Reduced apoptosis index rated by TUNEL assay; B) Expression of Bax, caspase- 3 and Bcl-2 protein in the neuronal tissue. 

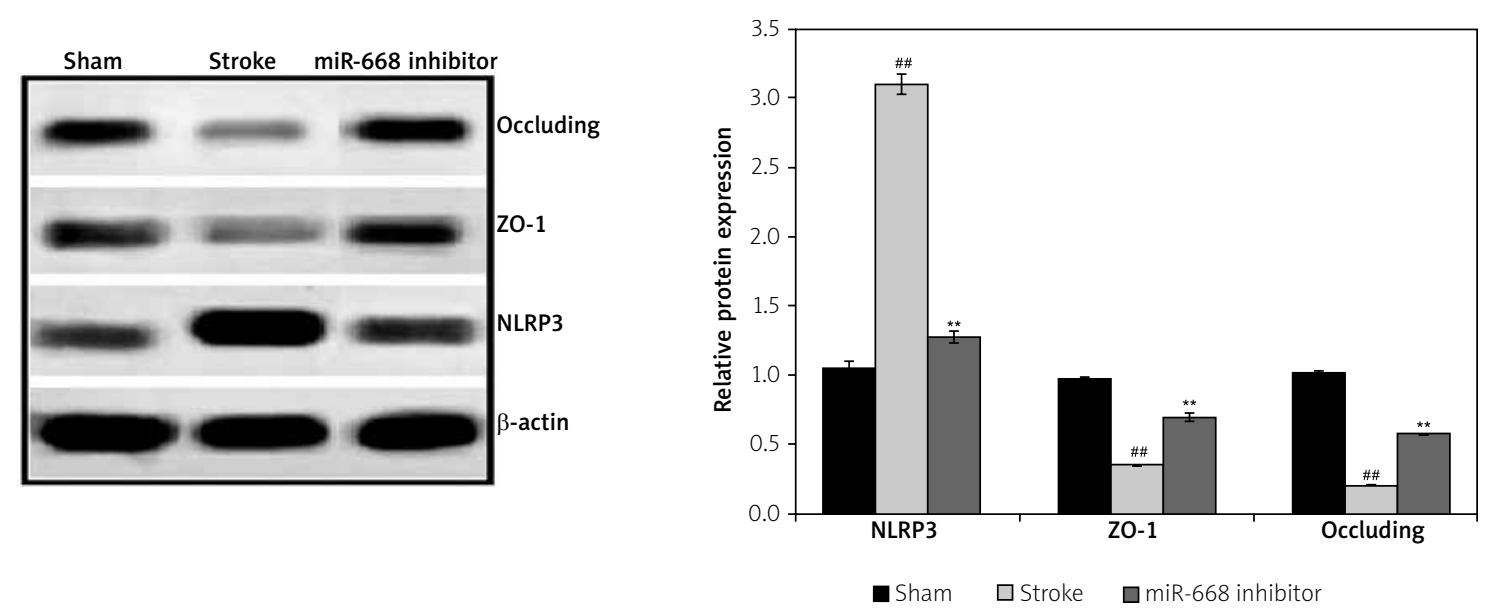

Fig. 6. miR-668 inhibitor attenuates the expression of ZO-1, NLRP3 and occluding protein in the I/R induced stroke rat model.

The level of NLRP3 expression was significantly increased, and those of occludin and ZO-1 protein were significantly reduced, in the brain tissue of the stroke group compared to the sham group. However, treatment with miR-668 inhibitor ameliorated the changes in ZO-1, NLRP3 and occludin protein expression levels in I/R-induced stroke rats.

\section{miR-668 inhibitor attenuates Drp1 signalling}

Drp1 protein phosphorylation was examined in the brain tissue of miR-668 inhibitor-treated I/R-induced stroke rats. The levels of p-Drp1/Drp1 protein expression were increased in the brain tissues of the stroke group compared to the sham group. However,

A

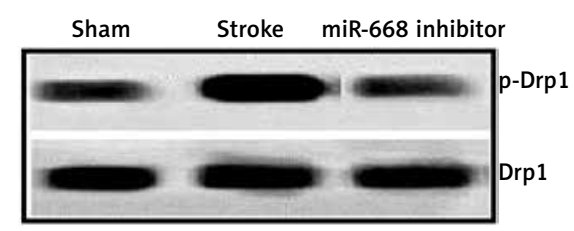

Drp1 expression was significantly reduced $(p<0.01)$ in the miR-668 inhibitor treatment group compared to the stroke group (Fig. 7A and B).

\section{Discussion}

Cerebral ischemia is a major cause of brain injury, and its management remains challenging. Therefore, we evaluated the protective effect of miR-668 inhibitor against I/R-induced stroke in terms of the neurological deficit score, cerebral infarct area and BBB permeability. In addition, the levels of inflammatory cytokines and expression of NLRP3, ZO-1, Drp1 and occludin proteins were estimated by ELISA and Western blotting, respectively. TUNEL assay and immunohistochemical analyses were performed to

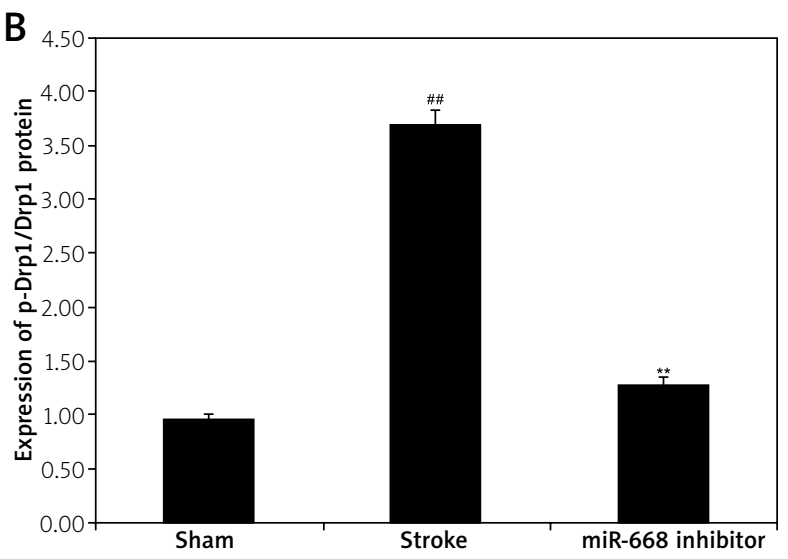

Fig. 7. miR-668 inhibitor attenuates the phosphorylation of Drp1 protein in the $I / R$ induced stroke rat model. A) Phosphorylation of Drp1 assessed by western blot assay; B) Expression of Drp1 assessed by immunohistochemical analysis. Mean $\pm \operatorname{SEM}(n=6),{ }^{\# \#} p<0.01$ compared to the sham group; ${ }^{* *} p<0.01$ compared to the stroke group. 
examine the effects of miR-668 inhibitor in I/R-induced stroke rats.

Ischemia/reperfusion-induced cerebral injury leads to disruption of the BBB, leading to secondary neuronal injury [24]. This suggests that ischemic brain disease progresses due to the linear relationship between cerebral I/R injury and disruption of the BBB [17]. Maintaining the integrity of the BBB prevents neuronal injury and improves neurological function [21]. The permeability of the BBB was reduced in our miR-668 inhibitor treatment group compared to the stroke group. Cerebral ischemia leads to an increase in the brain infarct area, which also reduces neurological function. Based on the neurological deficit score and infarct area, miR-668 inhibitor improved neurological function compared to the stroke group.

Tight junction proteins, such as ZO-1 and occlude, contribute to maintenance of the integrity of the BBB [22]. These proteins form a barrier that separates the cellular compartments and facilitates exchange of ions and other substances [8]. Altered ZO-1 and occludin protein levels may lead to changes in the integrity of the BBB. Tight junction proteins play a role in neuronal injury, and the expression of ZO-1 and occludin proteins protects against neuronal injury [7]. We found that treatment with miR-668 inhibitor normalised the altered expression levels of ZO-1 and occludin proteins in the cerebral tissues of I/R-induced stroke rats. I/R stimulates apoptosis, as indicated by the increased expression of a number of apoptosis-related proteins. The results of the present study indicated that inhibition of miR-668 ameliorated the alterations in Bax, caspase- 3 and $\mathrm{BCl}-2$ protein expression levels in the brain tissue of I/R-induced stroke rats.

Inflammatory cytokines contribute to the development of I/R-induced neuronal injury. The levels of these cytokines are increased by disruption of the $B B B$. The inflammatory response is enhanced by the activation of inflammasomes (NLRP3) in the cerebral I/R-induced stroke rat model [12]. In cerebral disease, NLRP3 contributes to disruption of the BBB, which leads to neuronal injury, whereas inhibition of NLRP3 prevents neuronal injury [1]. The expression of NLRP3 was reduced in the cerebral tissues of our miR-668 inhibitor-treated I/R-induced stroke rats.

I/R was previously shown to increase stress in the visceral organs, including the brain, by enhancing the production of ROS [9]; this was supported by our observations. A reduction in the ROS level is beneficial for the management of I/R-induced neuron injury. The results of the present study suggested that inhibition of miR-668 reduces the level of ROS in I/R-induced stroke rats. Activation of Drp1 protein was reported to contribute to mitochondrial fission, and phosphorylation of Drp1 was shown to regulate the mitochondrial membrane potential. I/R modulated the expression of Drp1 in the brain tissue, and treatment with miR-668 inhibitor reduced the phosphorylation of Drp1 protein in the brain tissue of I/R-induced stroke model rats.

\section{Conclusions}

This study showed that inhibition of miR-668 prevented neuronal apoptosis in a rat model of cerebral I/R-induced stroke, by modulating mitochondrial function and regulating NLRP3 signalling.

\section{Acknowledgements}

The authors are grateful to the Second Hospital of Hebei Medical University, China, for providing the necessary facilities to conduct this work.

\section{Disclosure}

The authors report no conflict of interest.

\section{References}

1. Bhattacharyya A, Chattopadhyay R, Mitra S, Crowe SE. Oxidative stress: an essential factor in the pathogenesis of gastrointestinal mucosal diseases. Physiol Rev 2014; 94: 329-354.

2. Chun N, Coca SG, He JC. A protective role for microRNA-688 in acute kidney injury. J Clin Invest 2018; 128: 5216-5218.

3. Costa V, Giacomello M, Hudec R, Lopreiato R, Ermak G, Lim D, Malorni W, Davies KJ, Carafoli E, Scorrano L. Mitochondrial fission and cristae disruption increase the response of cell models of Huntington's disease to apoptotic stimuli. EMBO Mol Med 2010; 2: 490-503.

4. Dong XX, Wang Y, Qin ZH. Molecular mechanisms of excitotoxicity and their relevance to pathogenesis of neurodegenerative diseases. Acta Pharmacol Sin 2009; 30: 379-387.

5. Guo X, Sesaki H, Qi X. Drp1 stabilizes p53 on the mitochondria to trigger necrosis under oxidative stress conditions in vitro and in vivo. Biochem J 2014; 461: 137-146.

6. Hu C, Huang Y, Li L. Drp1-dependent mitochondrial fission plays critical roles in physiological and pathological progresses in mammals. Int J Mol Sci 2017; 18: E144.

7. Jayaraj RL, Azimullah S, Beiram R, Jalal FY, Rosenberg GA. Neuroinflammation: friend and foe for ischemic stroke. J Neuroinflammation 2019; 16: 142 
8. Jiao H, Wang Z, Liu Y, Wang P, Xue Y. Specific role of tight junction proteins claudin-5, occludin, and ZO-1 of the blood-brain barrier in a focal cerebral ischemic insult. J Mol Neurosci 2011; 44: 130-139.

9. Jhun BS, O-Uchi J, Adaniya SM, Cypress MW, Yoon Y. Adrenergic regulation of Drpl-driven mitochondrial fission in cardiac phys io-pathology. Antioxidants (Basel) 2018; 7: E195.

10. Kalogeris T, Baines CP, Krenz M, Korthuis RJ. Cell biology of ischemia/reperfusion injury. Int Rev Cell Mol Biol 2012; 298 229-317.

11. Kelley N, Jeltema D, Duan Y, He Y. The NLRP3 inflammasome: an overview of mechanisms of activation and regulation. Int I Mol Sci 2019; 20: E3328.

12. Kuwar R, Rolfe A, Di L, Xu H, He L, Jiang Y, Zhang S, Sun D. A novel small molecular NLRP3 inflammasome inhibitor alleviates neuroinflammatory response following traumatic brain injury. J Neuroinflammation 2019; 16: 81.

13. Lima AR, Santos L, Correia M, Soares P, Sobrinho-Simões M, Melo M, Máximo V. Dynamin-related protein 1 at the crossroads of cancer. Genes (Basel) 2018; 9: E115.

14. McKeever PM, Schneider R, Taghdiri F, Weichert A, Multani N, Brown RA, Boxer AL, Karydas A, Miller B, Robertson J, Tartaglia MC. MicroRNA expression levels are altered in the cerebrospinal fluid of patients with young-onset Alzheimer's disease. Mol Neurobiol 2018; 55: 8826-8841.

15. Minutoli L, Puzzolo D, Rinaldi M, Irrera N, Marini H, Arcoraci V Bitto A, Crea G, Pisani A, Squadrito F, Trichilo V, Bruschetta D, Micali A, Altavilla D. ROS-mediated NLRP3 inflammasome activation in brain, heart, kidney, and testis ischemia/reperfusion injury. Oxid Med Cell Longev 2016; 2016: 2183026.

16. Ozaki E, Campbell M, Doyle SL. Targeting the NLRP3 inflammasome in chronic inflammatory diseases: current perspectives. J Inflamm Res 2015; 8: 15-27.

17. Ronaldson PT, Davis TP. Blood-brain barrier integrity and glial support: mechanisms that can be targeted for novel therapeutic approaches in stroke. Curr Pharm Des 2012; 18: 3624-3644.

18. Sekhon MS, Ainslie PN, Griesdale DE. Clinical pathophysiology of hypoxic ischemic brain injury after cardiac arrest: a "two-hit" model. Crit Care 2017; 21: 90

19. Soler EP, Ruiz VC. Epidemiology and risk factors of cerebral ischemia and ischemic heart diseases: similarities and differences. Curr Cardiol Rev 2010; 6: 138-149.

20. Sun Q, Fan J, Billiar TR, Scott MJ. Inflammasome and autophagy regulation - a two-way street. Mol Med 2017; 23: 188-195.

21. Stamatovic SM, Johnson AM, Keep RF, Andjelkovic AV. Junctional proteins of the blood-brain barrier: New insights into function and dysfunction. Tissue Barriers 2016; 4: e1154641.

22. Tapia R, Kralicek SE, Hecht GA. Modulation of epithelial cell polarity by bacterial pathogens. Ann N Y Acad Sci 2017; 1405 16-24.

23. Tsoi B, Chen X, Gao C, Wang S, Yuen SC, Yang D, Shen J. Neuroprotective effects and hepatorenal toxicity of Angong Niuhuang Wan against ischemia-reperfusion brain injury in rats. Front Pharmacol 2019; 10: 593.

24. Venkat P, Chopp M, Chen J. Blood-brain barrier disruption, vascular impairment, and ischemia/reperfusion damage in diabetic stroke. J Am Heart Assoc 2017; 6: e005819.
25. Wei Q, Sun H, Song S, Liu Y, Liu P, Livingston MJ, Wang J, Liang M, Mi QS, Huo Y, Nahman NS, Mei C, Dong Z. MicroRNA-668 represses MTP18 to preserve mitochondrial dynamics in ischemic acute kidney injury. J Clin Invest 2018; 128: 5448-5464.

26. Zhang Z, Liu S, Huang S. Effects of thymosin 4 on neuronal apoptosis in a rat model of cerebral ischemia-reperfusion injury. Mol Med Rep 2019; 20: 4186-4192.

27. Zhao XY, Lu MH, Yuan DJ, XU DE, Yao PP, Ji WL, Chen H, Liu WL, Yan CX, Xia YY, Li S, Tao J, Ma QH. Mitochondrial dysfunction in neural injury. Front Neurosci 2019; 13: 30. 